\title{
Effects of ion implantation on electron centers in hydrogenated amorphous carbon films
}

\author{
A. A. Konchits, M. Ya. Valakh, B. D. Shanina, S. P. Kolesnik, and I. B. Yanchuk ${ }^{\text {a) }}$ \\ Institute of Semiconductor Physics, Prospect Nauki 45, Kiev 03650, Ukraine \\ J. D. Carey and S. R. P. Silva \\ Advanced Technology Institute, School of Electronics and Physical Sciences, University of Surrey, \\ Guildford, GU2 7XH United Kingdom
}

(Received 27 June 2002; accepted 10 February 2003)

\begin{abstract}
Electron spin resonance (ESR) and Raman spectra measurements are carried out on $a-\mathrm{C}: \mathrm{H}$ and $a-\mathrm{C}: \mathrm{H}: \mathrm{N}$ films both as grown and implanted with $\mathrm{W}$ and $\mathrm{Ni}$ ions with doses ranged from 0.5 $\times 10^{15}$ to $1.2 \times 10^{16} \mathrm{~cm}^{-2}$. The as-grown films have small concentration of paramagnetic centers with a spin density $N_{s}$ of $10^{17} \mathrm{~cm}^{-3}$. Upon implantation a significant increase in $N_{s}$ of $(0.5-22)$ $\times 10^{19} \mathrm{~cm}^{-3}$ centers with $g_{(\mathrm{Si})}=2.0055$ and $g_{(\mathrm{C})}=2.0025$ was observed. These defects are ascribed to dangling bonds in the silicon substrate and in the carbon film, respectively. The correlation between variation of $N_{s}$ value with implantation dose and behavior of $D$ and $G$ band position and their intensity ratio in the visible Raman spectra is observed. The effects are attributed to changes in the $s p^{2}-s p^{3}$ systems and hydrogen loss due to ion induced annealing of the carbon films at high ion doses. The temperature and concentration dependencies of the ESR line shape and linewidth are explained using the mechanism of motional narrowing over the temperature range 4.2-300 K. Low temperature anisotropy of the $g$ value is found in the ESR spectra and is explained as arising from the dipole-dipole interaction in the infinitely thin films. (C) 2003 American Institute of Physics.
\end{abstract}

[DOI: 10.1063/1.1564280]

\section{INTRODUCTION}

Thin amorphous carbon films have attractive mechanical, optical, electrical, and tribological properties, and occupy an important place due to their wide industrial applications as thin film protective coatings. ${ }^{1}$ Hydrogenated amorphous carbon, $a-\mathrm{C}: \mathrm{H}$, is a useful material because of the possibility to vary the bonding configurations of the carbon atoms and to decrease film internal stresses. ${ }^{2,3}$ These properties of amorphous carbon films, which include hydrogenated diamond-like carbon (DLHC), may be modified by varying the deposition technology and/or by means of the introduction of various impurities during deposition or through implantation of ions after growth. ${ }^{4-7}$ The common feature of these films is the $\mathrm{C}$ bonding degeneracy, leading to the various concentration of nondiamond (mainly $s p^{2}$ ) bonded carbon. This fact has a significant influence not only on the film structure but also on the film electronic properties such as the width of the band gap and the density of states in $\mathrm{it}^{8}$ and consequently to the electric transport and optical properties. ${ }^{9}$ As a rule, the spin density in the band gap is often more then $10^{18} \mathrm{~cm}^{-3}$ in the $a-\mathrm{C}$ films ${ }^{10}$ and results in pinning of the Fermi level thus making it difficult to change electronic properties by doping.

Among the few methods of analyzing electron defect states, electron spin resonance (ESR) is a powerful direct method to the study of unpaired electron states in solids and has been employed in the study of amorphous carbon films produced by different methods. ${ }^{6-16}$ It provides important

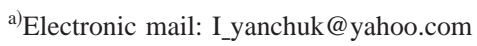

information about the spin density and nature of unpaired electrons in the films, their spin-spin and spin-lattice interactions, and linking of these parameters with films microstructure. ${ }^{9}$ In this article, we analyze the features of the ESR signal behavior in both nitrogenated and nitrogen-free $\mathrm{W}$ and Ni implanted $a$-C:H films. Visible Raman scattering measurements are also used to clarify the correlation between structure features and paramagnetic state properties.

\section{EXPERIMENTAL DETAILS}

The $a$-C:H films were deposited using a commercial rf $(13.56 \mathrm{MHz})$ plasma enhanced chemical vapor deposition (PECVD) system. The films were grown on the earthed electrode using $\mathrm{CH}_{4}$ and $\mathrm{He}$ as feed gases onto high resistivity $\mathrm{Si}$ (100) $(10-100 \Omega \mathrm{cm})$. Deposition on the earthed electrode results in a low negative self bias of $(<20 \mathrm{~V})$ and results in polymeric $a-\mathrm{C}: \mathrm{H}$ films with a high optical gap $(2.6 \mathrm{eV}){ }^{15}$ The hydrogen concentration in the initial films is $45-50$ at. $\%{ }^{17}$ Nitrogen containing samples were deposited with 10 sccm $\mathrm{N}$ that results in about 6 at. \% $\mathrm{N}$ contained within the film. Samples were implanted with $\mathrm{Ni}$ or $\mathrm{W}$ ions with doses in the range $(0.5-12) \times 10^{15} \mathrm{~cm}^{-2}$ at the University of Surrey ion beam center.

ESR measurements were performed in the X-band ESR spectrometer employed $100 \mathrm{kHz}$ field modulation (microwave frequency $\nu \cong 9.4 \mathrm{GHz}$ ) in the temperature range from 4.2 to $300 \mathrm{~K}$. The typical sizes of samples were $\sim 10 \times 5$ $\times d \mathrm{~mm}^{3}$, where $d$ is a thickness of film. The goal of filmon-substrate ESR measurements was to derive information 


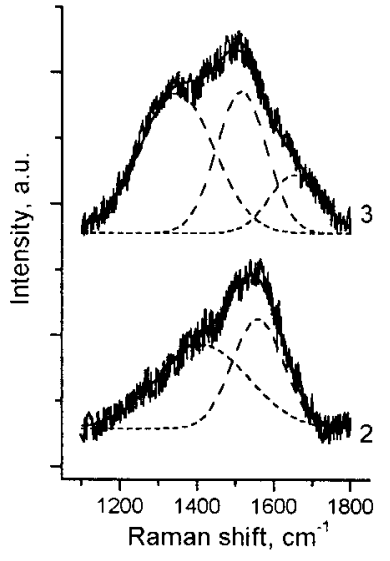

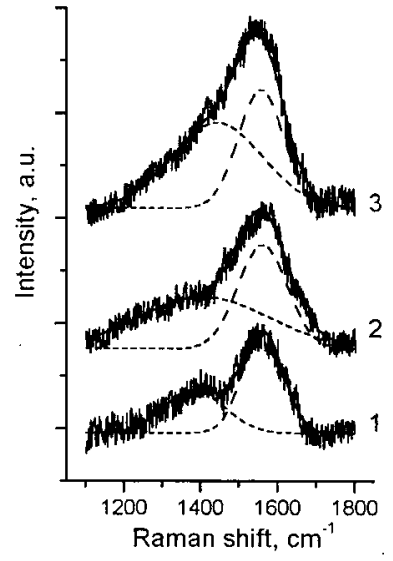

b
FIG. 1. Raman spectra of W implanted (a) $\alpha$-C:H:N samples and (b) $\alpha$-C:H for various doses: (1) $\phi=0.5 \times 10^{15}$, (2) $\phi=1.8 \times 10^{15}$, and (3) $\phi=7.5$ $\times 10^{15} \mathrm{~cm}^{-2}$.

about possible nonisotropic phenomena on ESR parameters. The estimated accuracy of the $g$-factor measurement is $\pm 10^{-4}$ for samples with $\Delta H_{\mathrm{pp}} \leqslant 5 \mathrm{G}$ and $\pm 2 \times 10^{-4}$ for $\Delta H_{\mathrm{pp}} \sim 10 \mathrm{G}$. The absolute accuracy of spin density estimation was $\pm 50 \%$ whereas the relative accuracy of $N_{s}$ measurements from sample-to-sample was $\pm 20 \%$. The Raman spectra were detected at RT using a double monochromator, a cooled photomultiplier employing standard photon counting technique and an $\mathrm{Ar}^{+}$laser at $\lambda=488 \mathrm{~nm}$ for excitation. For analysis all spectra were fitted with Gaussian lines. The data are presented after subtraction of the background due to photoluminescence.

\section{RESULTS}

\section{A. Raman spectra}

The Raman spectra from PECVD $a-\mathrm{C}: \mathrm{H}$ and $a-\mathrm{C}: \mathrm{H}: \mathrm{N}$ films with $D$ (at $\sim 1355 \mathrm{~cm}^{-1}$ ) and $G\left(\right.$ at $\sim 1581 \mathrm{~cm}^{-1}$ ) peaks were observed for the samples under study [see Figs. 1(a) and 1(b)]. The $G$ peak is usually assigned to zone center phonons of $E_{2 g}$ symmetry, ${ }^{18}$ while the $D$ peak, which is the breathing mode of $A_{1 g}$ symmetry, involves phonons near the $K$ zone boundary. The latter mode is forbidden in perfect graphite and becomes active only in presence of disorder. ${ }^{19}$ The Raman spectra intensity is very low due to the strong photoluminescence (PL) background signal from the nonimplanted samples. The intensities of the Raman peaks increase

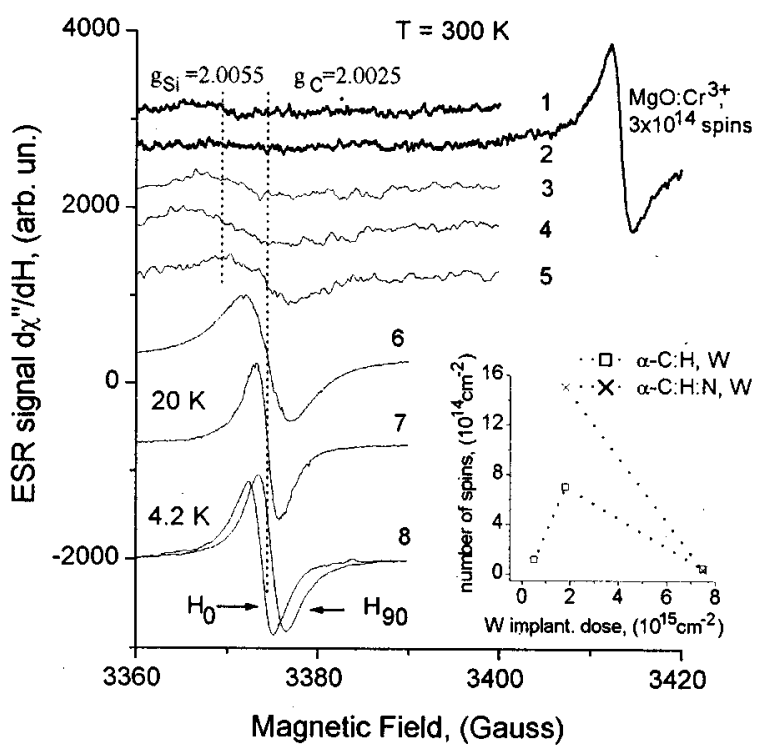

FIG. 2. The ESR spectrum on the initial and implanted samples $\alpha-\mathrm{C}: \mathrm{H}$, $\alpha$-C:H:N films; 1-G13, 2-G10, 3-W3N, 4-W3, 5-W1, 6, 7, 8-W2. All the spectra in this work reduced to $\nu=9.46 \mathrm{GHz}$. The inset shows spin density vs W implantation dose.

and the PL intensity decreases for the implanted samples. The Raman spectra were described by three Gaussian lines (usual $D, G$ peaks and peak at $\sim 1653 \mathrm{~cm}^{-1}$ ) for $a-\mathrm{C}: \mathrm{H}: \mathrm{N}$ at high $\mathrm{W}$ ion implantation dose [see Fig. 1(a)]. It was not possible to separate two bands in Raman spectra for Ni implanted samples.

\section{B. Electron spin resonance}

A summary of the room temperature ESR characteristics of the films studied in this work is reported in Table I. It is evident from Table I, that a significant concentration of paramagnetic centers (PC) is revealed for all implanted samples, while as grown and nonimplanted samples G10 and G13 (without and with $\mathrm{N}$ ) have only small PC concentration $N_{s}$ $\leqslant 10^{17} \mathrm{~cm}^{-3}$. Figure 2 shows the ESR spectra for the unimplanted samples and for samples, which were implanted by $\mathrm{W}$ ions. It was found, that each of the weak signals in curves $1,3-5$ at $T=300 \mathrm{~K}$ could be described as a sum of two ESR signals with $g$-factors $g=2.0055$ and 2.0025 . The contribution of the signal with $g=2.0025$ increases as the total intensity of ESR spectrum grows. In fact, the spectrum for

TABLE I. Characteristics of the studied samples. $N_{s, \text { sum }}$ is a total spin density; $N_{s 1 \text {,sum }}$ is a number of spins per $\mathrm{cm}^{2}$.

\begin{tabular}{|c|c|c|c|c|c|c|c|c|c|}
\hline Sample & G10 & G13 & W1 & W2 & $\begin{array}{c}\mathrm{W} 2 \\
\mathrm{~N}\end{array}$ & W3 & $\begin{array}{l}\text { W3 } \\
\mathrm{N}\end{array}$ & $\begin{array}{c}\mathrm{Ni} \\
1\end{array}$ & $\begin{array}{c}\mathrm{Ni} 1 \\
\mathrm{~N}\end{array}$ \\
\hline $\mathrm{N}$ flow, sccm & $\cdots$ & 10 & $\cdots$ & $\cdots$ & 10 & $\ldots$ & 10 & $\ldots$ & 10 \\
\hline Implantation ion & $\cdots$ & $\cdots$ & $\mathrm{W}$ & $\mathrm{W}$ & $\mathrm{W}$ & $\mathrm{W}$ & W & $\mathrm{Ni}$ & $\mathrm{Ni}$ \\
\hline Dose, $10^{15} \mathrm{~cm}^{-2}$ & 0 & 0 & 0.5 & 1.8 & 1.8 & 7.5 & 7.5 & 12 & 12 \\
\hline$N_{s l, \mathrm{sum}}, 10^{14} \mathrm{~cm}^{-2}$ & $<0.03$ & $\sim 0.03$ & 1.5 & 10 & 22 & 0.7 & 0.5 & 21 & 15 \\
\hline$N_{s, \text { sum }}, 10^{19} \mathrm{~cm}^{-3}$ & $<0.01$ & $\sim 0.01$ & 1.5 & 10 & 22 & 0.7 & 0.5 & 21 & 15 \\
\hline$D$ band position, $\mathrm{cm}^{-1}$ & $\ldots$ & $\ldots$ & 1383 & 1409 & 1415 & 1443 & 1348 & $\ldots$ & $\ldots$ \\
\hline$G$ band position, $\mathrm{cm}^{-1}$ & $\cdots$ & $\cdots$ & 1561 & 1561 & 1558 & 1556 & 1515 & $\cdots$ & $\cdots$ \\
\hline$I(D) / I(G)$ & $\cdots$ & $\cdots$ & 0.65 & 1.33 & 1.36 & 1.44 & 1.44 & $\cdots$ & $\cdots$ \\
\hline
\end{tabular}




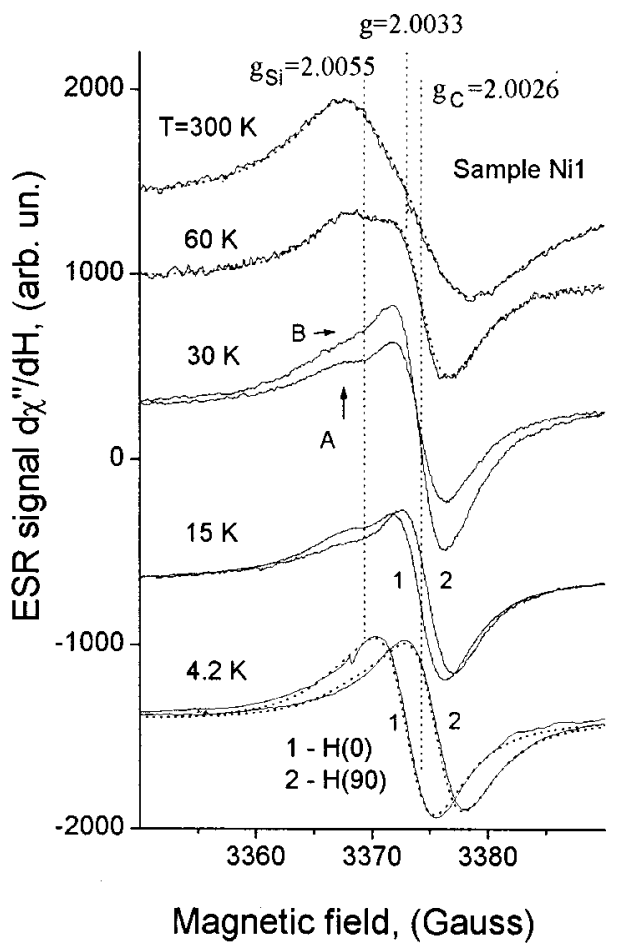

FIG. 3. The ESR spectrum on the sample Ni1 at different temperatures. A, $\mathrm{B}-P_{\mathrm{mw}}=10$ and $2 \mathrm{~mW}$, respectively. Dotted lines are shown fit sum of two Lorentzians (at $T=300$ and $60 \mathrm{~K}$ ) and single Lorentzian (at $T=4.2 \mathrm{~K}$ ) as well.

samples W2 and W2N consists totally of the signal with $g$ $=2.0025$ (see Fig. 2). The shape of the spectrum changes from a distorted super-Lorentzian curves (curves 1,3-5) to a pure Lorentzian signal shape for samples with the intense signal (curve 6). The maximum concentration of PC is achieved for samples $\mathrm{W} 2$ and $\mathrm{W} 2 \mathrm{~N}$ with the intermediate implantation dose (see inset at Fig. 2).

The ESR spectra for the sample Nil (implanted with $\mathrm{Ni}$ ) are presented in Fig. 3. The ESR spectrum of this sample at room temperature can be well described by a single Lorentzian curve with $g=2.0033$, or equally as well as the sum of two Lorentzian lines with a different width, with $g=2.0055$ and $g=2.0026$ and weight factors $\sim 0.25$ and 0.75 , respectively. The analysis of this spectrum at $T=60 \mathrm{~K}$ shows, that the spectrum presents the combination of two Lorentzian lines with the above $g$ factors (see dotted line on the Fig. 3 at $T=60 \mathrm{~K}$ ). The reason of the spectrum splitting at $T=60 \mathrm{~K}$ is explained by the different saturation behavior with temperature of its components. As a result, the total spectrum at $T$ $\leqslant 30 \mathrm{~K}$ is characterized by the dependence of the various line shapes on the applied microwave power $\left(P_{\mathrm{mw}}\right)$ (see Fig. 3). At $T=4.2 \mathrm{~K}$ and $P_{\mathrm{mw}} \sim 2 \mathrm{~mW}$ the spectrum component with $g=2.0055$ is saturated completely and only a single Lorentzian line with $g=2.0026$ is observed (the low-temperature $g$ anomalies will be described further). Nitrogen containing sample Ni1N exhibits a similar behavior. The temperature dependences of the ESR linewidth for the implanted samples are shown in Fig. 4. It can be observed from curves 1 and 2, that samples W3 and W3N, with a small concentration of PC give the signals where $\Delta H_{\mathrm{pp}}$ remains constant as the temperature increases. However, the rate by which $\Delta H_{\mathrm{pp}}$ in-

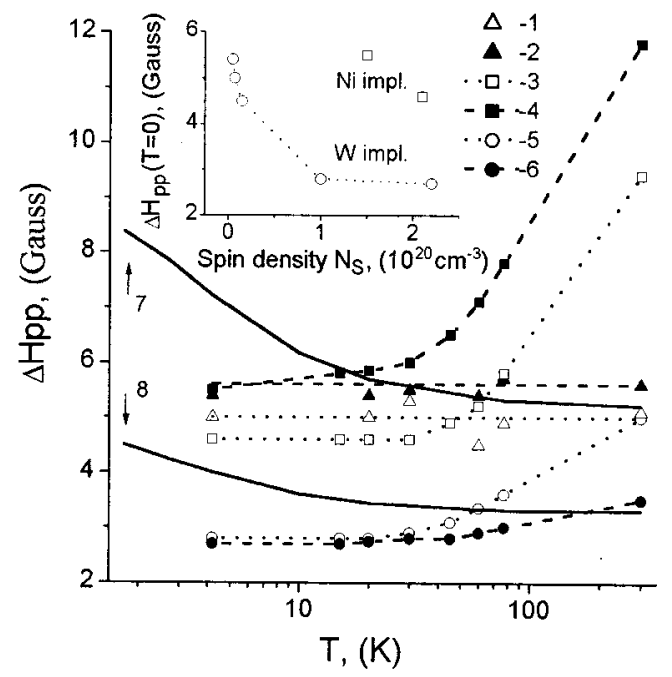

FIG. 4. Variations of linewidth of ESR signals as a function of the measurement temperature; 1-W3, 2-W3N, 3-Ni1, 4-Ni1N, 5-W2, 6-W2N. Curves 7, 8-DLHC-1 (deposition $\mathrm{CH}_{4}$ ) and DLHC-2 (deposition $\mathrm{C}_{2} \mathrm{H}_{2}$ ), respectively (see Ref. 16). The inset shows the ESR linewidth vs spin density for the implanted samples. It is seen the act of the motion narrowing mechanisms.

creases with temperature is higher for samples with a higher concentration $N_{s}$. Over the entire temperature range samples implanted with $\mathrm{Ni}$ have a higher value of linewidth than those implanted with $\mathrm{W}$ (curves 3, 4 and 5, 6, respectively). For a comparison, values of $\Delta H_{\mathrm{pp}}(T)$ for nonimplanted samples DLHC-1 and DLHC- ${ }^{16}$ with smaller content of hydrogen (curves 7,8 ) are also presented. In the latter case, $\Delta H_{\mathrm{pp}}$ decreases as the temperature increases.

It was also observed that as the temperature was decreased, the $g$ value became anisotropic. Figure 2 (curve 8) and Fig. 3 show this phenomenon for some $\mathrm{W}$ and $\mathrm{Ni}$ implanted samples, where $H_{0}$ and $H_{90}$ are the orientations of magnetic field along and orthogonal to the substrate plane, respectively. The changes in $g_{0}$ and $g_{90}$ as a function of temperature are shown in Fig. 5 where it is seen that the value of $\Delta g=g_{(0)}-g_{(90)}$ decreases with temperature. Using the data in Table I for PC it is possible to conclude that the largest value of $\Delta g$ is observed in those samples with the having the larger concentration of paramagnetic centers. The inset to Fig. 5 shows the values of $\Delta g$ as a function versus $N_{s}$. The dependence $\Delta g\left(N_{s}\right)$ for nonimplanted DLC films ${ }^{16}$ is also presented for comparison. One can see that at the same spin density $\Delta g$ for the implanted samples is larger.

\section{ANALYSIS AND DISCUSSION}

\section{A. Raman spectra behavior}

An intensive photoluminescence is observed for the nonimplanted samples and implies a significant content of hydrogen $(\geqslant 40 \text { at. } \%)^{20}$ and rather high $s p^{3}$ bond content. The structure of these samples can be characterized by the presence of polymeric bonding type due to saturation of $\mathrm{C}=\mathrm{C}$ bonds by $\mathrm{H}$ as $\equiv \mathrm{CH}_{x}$ groups, rather than by increasing the fraction of $\mathrm{C}-\mathrm{C}$ bonds. The peak positions of PL $(h \omega$ 


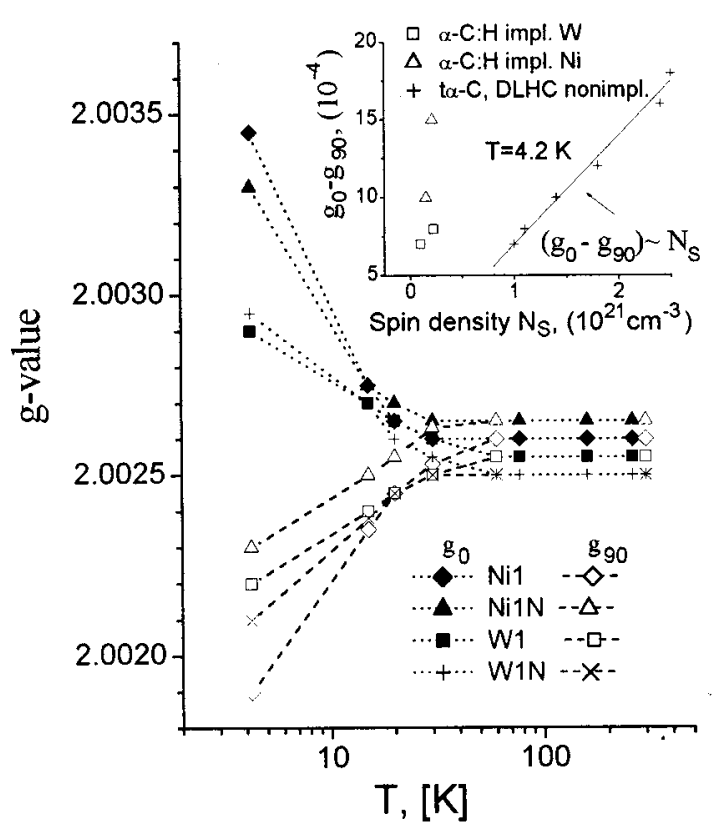

FIG. 5. The temperature dependence of $g_{(0)}$ and $g_{(90)}$ for the implanted films. The insertion on figure shows the dependence of $g$-anisotropy $\Delta g$ $=g_{(0)}-g_{(90)}$ on PC concentration both in the implanted samples and some as deposited DLC films with high PC concentration at $T=4.2 \mathrm{~K}$.

$\approx 2.6 \mathrm{eV}$ ) is indicative of a high $s p^{3}$ content, estimated to be near $80 \%,{ }^{18}$ which results in very low intensity ratio $I(D) / I(G) .^{19}$

As a result of implantation with $\mathrm{W}$ ions there are some effects: intensive hydrogen loss, the reorganization of the $s p^{3}$ and $s p^{2}$ phases and the creation of radiation defects. ${ }^{21,22}$ The redshift of the PL band due to the Tauc Gap decreasing ${ }^{19}$ confirms the first effect is prevalent. All of these factors are related with reduction of $s p^{3}$ content in the films. On the basis of the analysis presented in Ref. 18 we estimate the $s p^{3}$ content to be $0.35-0.45$ for a $G$-peak position near 1560 $\mathrm{cm}^{-1}$. Therefore, it may be supposed from these results, that the intensity ratio $I(D) / I(G)$ should be increased up to $0.6-$ 0.7 , consistent with our findings (see Table I). The results ${ }^{17}$ show that the implantation of $a-\mathrm{C}: \mathrm{H}$ samples with $\mathrm{C}$ ions by the dose $\sim 10^{16} \mathrm{~cm}^{-2}$ reduces the $\mathrm{H}$ concentration from 50 to $\sim 12$ at. $\%$. Therefore, one can expect that in our case the implantation by heavier $\mathrm{Ni}$ and $\mathrm{W}$ ions with similar dose would diminish hydrogen content at least to the same level.

When the ion dose increases, the radiation disordering process begins to dominate over hydrogen desorption. This is confirmed by the upshift of the $D$-peak position, which indicates disordering ${ }^{18}$ and by the increase of the $G$-peak width, which is proportional to the bond-angle disorder at $s p^{2}$ sites. ${ }^{19}$ Since the PL band position is not changed, we suggest, that the optical gap and, consequently, $s p^{3}$ content are not varied. Therefore, the $G$-peak position does not vary also $\left(1558 \mathrm{~cm}^{-1}\right.$ for $a-\mathrm{C}: \mathrm{H}: \mathrm{N}$ and $1561 \mathrm{~cm}^{-1}$ for $\left.a-\mathrm{C}: \mathrm{H}\right)$. In the case of nitrogen free films at high dose of $\mathrm{W}$ ion implantation we observed similar situation.

The situation for $a-\mathrm{C}: \mathrm{H}: \mathrm{N}$ films is more sophisticated. The high-frequency shoulder at $\sim 1653 \mathrm{~cm}^{-1}$ appears in spectra of these films. This peculiarity attributed to the ole- finic $\mathrm{C}=\mathrm{C}$ stretching mode. ${ }^{23,24}$ Also we observed that $I(D) / I(G)$ increases and indicates that the film have still sufficiently content of sixfold aromatic rings. Thus, in these films the transformation of the aromatic rings to the olefinic groups $^{19}$ and relaxation $s p^{3}$ dandling bonds to a $\mathrm{C}=\mathrm{C} s p^{2}$ configuration $^{25}$ take place. It is believed, that nitrogen encourages the existing $s p^{2}$ sites to clusters ${ }^{26}$ and causes the $s p^{2}$ sites graphitization. ${ }^{22}$ We suggest, that $\mathrm{N}$ stimulates the conversion the $s p^{3}$ dandling bonds to $s p^{2}$ aromatic rings and changes the $s p^{2}$ configuration from rings to short chains (olefins). The behavior of $D$-peak position verifies this transformation (see Table I). The low $G$-peak position can be explained by the mixing of $s p^{2}$ modes with lower-frequency $s p^{3}$ modes.

\section{B. Electron spin resonance}

In the as deposited samples G10 and G13 (with and without $\mathrm{N}$ ) there is a small concentration of paramagnetic defects $N_{s} \sim 10^{17} \mathrm{~cm}^{-3}$ (Fig. 2, curves 1, 2). Moreover, the ESR signal from sample G13 is caused, mainly, by the defects with $g=2.0055$ and is attributed to the dangling bonds from the Si substrate. ${ }^{27}$ This signal disappears after etching of substrate surface. The absence of $\alpha-\mathrm{C}$ related ESR signal is a remarkable result in sense of the passivation of defects due to hydrogen, and this fact indicates also, that the samples G10 and G13 are rather soft PLHC with low internal stresses. It was proposed in Ref. 10 for $t \alpha-\mathrm{C}$ and DLHC films, the concentration of defects and width of their ESR lines $\Delta H_{\mathrm{pp}}$ are moderated in accordance with a decrease of internal stresses. On the other hand, it was observed in Ref. 3 , that the DLHC films with concentration of hydrogen $c_{\mathrm{H}}$ $\sim 32$ at. $\%$ are rather soft PLHC films with a small level of the internal stresses, and in Ref. 11 it was revealed, that spin density is only $\sim 1.5 \times 10^{17} \mathrm{~cm}^{-3}$.

Analysis of the results in Figs. 2 and 3 show that the implantation causes the appearance of paramagnetic centers in the $a-\mathrm{C}$ film (defects with $g=2.0025),{ }^{6,9-16,27}$ and in the Si substrate $(g=2.0055) .{ }^{28}$ At small and at large W implantation doses the density of spins in the film and in the substrate are comparable (Fig. 2. curves 3-5). At the intermediate dose of W the ESR signal in film is the most intense and the signal from substrate with $g=2.0055$ is now undetectable (Fig. 2, curve 6). In the case of $\mathrm{Ni}$ implantation the signal from substrate is stronger and contributes $\sim 0.25$ to total intensity of signal (Fig. 3). These effects can be readily explained in terms of the lighter $\mathrm{Ni}$ ions being able to penetrate further into the $\mathrm{Si}$ substrate than the $\mathrm{W}$ ions. The presence of nitrogen in samples only weakly influences to the defect concentration.

The temperature and concentration dependencies of the ESR line shape and linewidth are due to the basic interactions between paramagnetic centers, when their concentration is large. Theoretical calculations of these parameters were performed in Refs. 10 and 16 in DLC that takes into account of both temperature dependent and temperature independent on interactions via

$$
\Delta H_{\mathrm{pp}}=\Delta H_{\mathrm{pp}}(c, T=0)+\Delta H_{\mathrm{pp}}(c, T)+\Delta H_{\mathrm{pp}}\left(c_{\mathrm{H}}, T\right),
$$


where $\Delta H_{\mathrm{pp}}(c, T=0)$ is the contribution to the linewidth from interactions which are independent of temperature, the latter including are DD and exchange interactions (EXI); $c$ $=N_{s} / N_{0}$ is a relative spin concentration, $N_{0}$ is the number of carbon atoms per $\mathrm{cm}^{-3}$ and $c_{\mathrm{H}}$ is the concentration of hydrogen. The first term in Eq. (1) can be written as the function of the second and the fourth moments of the ESR line, according to $^{29}$

$$
\Delta H_{\mathrm{pp}}(c, 0)=(\pi / 2 \sqrt{ } 3) \times m_{2}^{3 / 2} \times\left[m_{4}+(\pi / 2) \times m_{2} \times \omega_{e}^{2}\right]^{-1 / 2},
$$

where $\quad m_{2}=3.8 \times g^{2} \times \beta 2 \times N_{0}^{2} \times c ; \quad m_{4}=3 m_{2}^{2} \times(0.63$ $+0.068 / c)$ and $\omega_{e}^{2}=2.12 J_{0}^{2} \times \exp \left(-\alpha / c^{1 / 3}\right)$; where $\alpha$ $=2 d_{0} / R_{0}, d_{0}$ is a radius of the volume per a host atom, such that $(4 \pi / 3) \times d_{0}^{3} \times N_{0}=1$, and $d_{0}=1.34 \AA$. $R_{0}$ is a radius of the wave function of the PC and $J_{0}$ is a constant of EXI between the nearest paramagnetic centers. Equation (2) reflects the known phenomenon of the narrowing of ESR line with concentration growth due to EXI between identical centers. The dependence of Eq. (2) on PC concentration $N_{s}$ presents a curve with a minimum, which is achieved at concentration $N_{s} \sim 10^{21} \mathrm{~cm}^{-3}$ for PC in DLC films. ${ }^{16}$ The dependence $\Delta H_{\mathrm{pp}}\left(N_{s}, 0\right)$ for our $\mathrm{W}$ implanted samples (see insert to Fig. 4) also demonstrates the role of the exchange narrowing but the minimum $\Delta H_{\mathrm{pp}}\left(N_{s}, 0\right)$ occurs at a lower concentration $\left(2.2 \times 10^{20} \mathrm{~cm}^{-3}\right)$. This is readily explained by the inhomogeneous distribution of defects in the implanted samples, when the local concentration of PC $N_{s, \text { loc }}$ is significantly higher than the average measured one. The observation of a Lorentzian line shape for samples W2 (Fig. 2, curves $6-8$ ) and Ni1 (Fig. 3 at $T=4.2 \mathrm{~K}$ ) with high concentration of spins is in accordance with the conclusion about important role of the exchange narrowing of ESR signal.

The presence of hydrogen in the films causes an appearance of the hyperfine interaction between the residual protons and the PC and gives an additive contribution to $\Delta H_{\mathrm{pp}}{ }^{6,15,16,30}$ In Ref. 15 it was found the empirical relation between the peak-to-peak Gaussian contribution and the hydrogen content at room temperatures: $\Delta H_{\mathrm{pp}}^{G}$ (in Gauss) $=(0.18 \pm 0.05) \times$ at. $\% \mathrm{H})$. In Ref. 16 it was established that the influence of hydrogen can be included to mechanism of exchange and motion narrowing and depends on the concentration of PC and on the temperature. For comparison the curves 7, 8 on the Fig. 4 shows the temperature dependence of $\Delta H_{\mathrm{pp}}{ }^{16}$ for the samples with intermediate (DLHC-1, curve 7) and small (DLHC-2, curve 8) content of hydrogen. These dependences demonstrate that $\Delta H_{\mathrm{pp}}$ decrease with temperature increase due to motion narrowing (electron jumps between centres with different resonance fields). The influence of hydrogen to $\Delta H_{\mathrm{pp}}$ grows with growing of hydrogen concentration $c_{\mathrm{H}}$ is in a qualitative accordance with conclusions reached in Ref. 15. In our implanted samples with high concentration of hydrogen an opposite behavior of $\Delta H_{\mathrm{pp}}$ is observed (Fig. 4, curves 1-6), e.g., hydrogen does not cause the linewidth change for samples W2 and W2N (curves 5 and 6), as it would in hydrogen free samples. It is not surprising, since the implantation processes are accompanied hydrogen effusion. ${ }^{17,31}$ The role of hydrogen in the samples with a small concentration of PC comes to the broadening of
$\Delta H_{\mathrm{pp}}$, along with DD interaction, and gives relatively wide and independent on temperature ESR line (Fig. 4, curves $1-2)$. There is also an indirect mechanism of hydrogen influence on the concentration of PC by means of intrinsic stress change in films. This influence and also hydrogen effusion process during implantation make clear the irregular dependence of PC concentration $N_{s}$ versus implantation dose (see insert in Fig. 2).

Concerning $\Delta H_{\mathrm{pp}}(T)$ for the nickel implanted samples (Fig. 4, curves 3, 4) we note that the RT linewidth is twice the width at low temperature and a more strong dependence with temperature increase to be compared with $\mathrm{W}$ implanted samples at the same PC concentration (Fig. 4, curves 5, 6). There is the additive contribution to $\Delta H_{\mathrm{pp}}$ due to spin-spin $\left(T_{2}^{-1}\right)$ and spin-lattice $\left(T_{1}^{-1}\right)$ relaxation through the hyperfine interaction of PC electron with paramagnetic nuclei of $\mathrm{Ni}$ or through the spin-orbital interaction with valence electrons of $\mathrm{Ni}$, but this question is not considered in this article.

The values $g=2.0025$ (1) for the $\mathrm{W}$ implanted samples and $g=2.0026(1)$ for the $\mathrm{Ni}$ implanted samples at $T$ $=300 \mathrm{~K}$ precisely coincides with those for nonimplanted samples $t \alpha-\mathrm{C}$ and DLHC films. ${ }^{10,16}$ However, the low temperature resonance magnetic field ( $g$-value) anisotropy (see Fig. 5) is one of the most interesting phenomenon. It was observed in $t \alpha-\mathrm{C}$ films at low temperature in Ref. 10 and was attributed ${ }^{10,16}$ to DD interaction in the infinitely thin films, which becomes significant at high density of the centres and at low temperature. In this way the magnetic anisotropy is due to the form factors of quasitwo-dimensional DLC films or thin large area $s p^{2}$ clusters. In the present experiments we have observed a similar behavior, when $\Delta g$ value is proportional to spin density $N_{s}$ and to $T^{-1}$ (see Fig. 5 and inset). A simple comparison with Refs. 10 and 16 demonstrates, that for $N_{s}$ of $2 \times 10^{20} \mathrm{~cm}^{-3}$ and $T=4.2 \mathrm{~K}$ the magnitude $\Delta g$ should not exceed $2 \times 10^{-4}$, while experiments with implanted samples we have observed $\Delta g=(10-15)$ $\times 10^{-4}$ (see Fig. 5). It is clear that the resonance field is formed by the local concentration of PC and $\Delta g$ observed is due to the quasitwo-dimensional character of sample fragments, which do not interact each to other magnetically.

\section{CONCLUSIONS}

We have studied the effects on the ESR and Raman spectra of $\mathrm{Ni}$ and $\mathrm{W}$ implantation in PECVD $a-\mathrm{C}: \mathrm{H}$ and $a-\mathrm{C}: \mathrm{H}: \mathrm{N}$ films. The ion implantation essentially reduces a content of hydrogen and initiates the radiation defects. In the case of nitrogen free films the radiation disordering increases with increase of ion dose. We observed the ion induced annealing for $a-\mathrm{C}: \mathrm{H}: \mathrm{N}$ films at high $\mathrm{W}$ ion dose due to changes in the $s p^{2}-s p^{3}$ phases. The ESR measurements show that the as grown films contain a low density of spins $N_{s}<10^{17} \mathrm{~cm}^{-3}$. The implantation of $0.5 \times 10^{15}-1.2 \times 10^{16}$ $\mathrm{cm}^{-2} \mathrm{~W}$ or $\mathrm{Ni}$ ions in films results in a significant concentration of paramagnetic defects $N_{s}$ in the films ( $g$ $=2.0025)$, as well as in the Si substrate $(g=2.0055)$, which are attributed to carbon and silicon related defects, respectively. The value of $N_{s}$ is not found to be proportional to the ion implantation dose in a case of $\mathrm{W}$ ion implantation, when 
the maximum value $N_{s} \cong 2 \times 10^{20} \mathrm{~cm}^{-3}$ is achieved at the intermediate implantation dose $\phi=1.8 \times 10^{15} \mathrm{~cm}^{-2}$. These results correlate with the data of Raman spectroscopy, pointing to a closed relation between the structure disordering and spin density in the implanted films.

The temperature dependence of the linewidth in the implanted samples has a character opposite to the usually observed for DLHC samples. The main cause of this behavior is the sharp decreasing of the hydrogen concentration by the implantation process in the samples with high dose implantation. The hydrogen may be present with essential concentration in the samples with lowest implantation doses results in the efficient mechanism of motion narrowing of the EPR line in samples with high $\mathrm{PC}$ concentration $\left(N_{s}\right.$ $\cong 10^{20} \mathrm{~cm}^{-3}$ ) even at low temperature. The unexpected effect of $g$-factor anisotropy is revealed here for amorphous paramagnetic materials of low temperature. It is explained by the anisotropy and concentration dependence of DD interaction between paramagnetic centers in thin films.

${ }^{1}$ J. L. Davidson, in Synthetic Diamond, Emerging CVD Science and Technology, edited by K. E. Spear, J. P. Dismukes (Wiley, New York, 1994).

${ }^{2}$ J. Fallon, V. S. Veerasamy, C. A. Davis, J. Robertson, G. A. J. Amaratunga, W. I. Milne, and J. Koskinen, Phys. Rev. B 48, 4777 (1993); J. Robertson, Philos. Mag. B 76, 335 (1997).

${ }^{3}$ J. Tersoff, Phys. Rev. 44, 12039 (1991); X.-M Tang, J. Weber, Y. Baer, C. Müller, W. Hänni, and H. E. Hintermann, Phys. Rev. B 48, 10124 (1993).

${ }^{4}$ J. Robertson and C. A. Davis, Diamond Relat. Mater. 4, 441 (1995).

${ }^{5}$ V. G. Litovchenko, A. A. Evtukh, R. I. Marchenko, N. I. Klui, and V. A. Semenovich, Appl. Surf. Sci. 111, 213 (1997).

${ }^{6}$ S. R. P. Silva, J. Robertson, G. A. J. Amaratunga, B. Rafferty, L. M. Brown, J. Schwan, D. F. Eranceschini, and G. Mariotto, J. Appl. Phys. 81, 2626 (1997).

${ }^{7}$ B. Druz, Y. Yevtukhov, V. Novotny, I. Zaritsky, V. Kanarov, V. Polyakov, and A. Rukavishnikov, Diamond Relat. Mater. 9, 668 (2000).
${ }^{8}$ U. Stephan, Th. Fraenheim, P. Blaudeck, and G. Jungnickel, Phys. Rev. B 50, 1489 (1994).

${ }^{9}$ R. U. A. Khan, J. D. Carey, S. R. P. Silva, B. J. Jones, and R. C. Barklie, Phys. Rev. B 63, 121201(R) (2001).

${ }^{10}$ B. Druz et al., Mater. Res. Soc. Symp. Proc. 593, 249 (2000).

${ }^{11}$ A. Sadki, Y. Bounouh, M. L. Theye, J. von Bardeleben, J. Cernogora, and J. L. Fave, Diamond Relat. Mater. 5, 439 (1996).

${ }^{12}$ R. C. Barklie, M. Collins, J. Cunniffe, and S. R. P. Silva, Diamond Relat. Mater. 7, 864 (1998).

${ }^{13}$ M. Fanciulli, G. Fusco, and A. Tagliaferro, Diamond Relat. Mater. 6, 725 (1997).

${ }^{14}$ G. Fusco, A. Tagliaferro, W. I. Milne, and J. Robertson, Diamond Relat. Mater. 6, 783 (1997).

${ }^{15}$ R. C. Barklie, M. Collins, and S. R. P. Silva, Phys. Rev. B 61, 3546 (2000).

${ }^{16}$ B. Druz et al., Diamond Relat. Mater. (to be published).

${ }^{17}$ R. U. A. Khan and S. R. P. Silva, Diamond Relat. Mater. 10, 224 (2001).

${ }^{18}$ M. A. Tamor and W. C. Vassell, J. Appl. Phys. 76, 3823 (1994).

${ }^{19}$ A. C. Ferrari and J. Robertson, Phys. Rev. B 61, 14095 (2000).

${ }^{20}$ B. Marchon, J. Gui, K. Grannen, G. C. Rauch, J. W. Ager, S. R. P. Silva, and J. Robertson, IEEE Trans. Magn. 33, 3148 (1997).

${ }^{21}$ I. Sela, M. Adel, and R. Beserman, J. Appl. Phys. 68, 70 (1990).

${ }^{22}$ J. Robertson, Phys. Status Solidi A 186, 177 (2001).

${ }^{23}$ S. Liu, S. Gangopadhyay, G. Sreenivas, S. S. Ang, and H. A. Naseem, Phys. Rev. B 55, 13020 (1997).

${ }^{24}$ K. Ogata, J. Fernando, D. Chubaci, and F. Fujimoto, J. Appl. Phys. 76, 3791 (1994)

${ }^{25}$ G. A. J. Amaratunga, J. Robertson, V. S. Veerasamy, W. I. Milne, and D. R. McKenzie, Diamond Relat. Mater. 4, 637 (1995).

${ }^{26}$ J. Robertson, Mater. Sci. Eng., R. 37, 129 (2002).

${ }^{27}$ Amorphous Semiconductors, Topics in Applied Physics 36, edited by M. H. Brodsky (Springer, Berlin, 1979).

${ }^{28}$ B. J. Jones, R. C. Barklie, R. U. A. Khan, J. D. Carey, and S. R. P. Silva, Diamond Relat. Mater. 10, 993 (2001).

${ }^{29}$ A. Abragam, The Principles of Nuclear Magnetism (Clarendon, Oxford, 1961).

${ }^{30}$ M. Hoinkis, E. D. Tober, R. L. White, and M. S. Crowder, Appl. Phys. Lett. 61, 2653 (1992).

${ }^{31}$ M. E. Adel, R. Kalish, and S. Prawer, J. Appl. Phys. 62, 4096 (1987). 